\title{
Persistent idiopathic facial pain associated with borderline personality disorder: a case report
}

\author{
Hugo André de Lima Martins ${ }^{1}$ (D) Bruna Bastos Mazullo Martins ${ }^{1}$ (D) Camilla Cordeiro dos Santos ${ }^{2}$ \\ Djanilson Jose Pontes² (D) Daniella Araújo de Oliveira ${ }^{3}$ (D) Marcelo Moraes Valenca ${ }^{4,5}$ (D)
}

${ }^{1}$ Unidade do Cérebro, Surubim, Pernambuco, Brazil.

${ }^{2}$ Esuda Faculty, Recife, Pernambuco, Brazil.

${ }^{3}$ Departmente of Physiotherapy, Universidade Federal e Pernambuco, Recife, PE, Brazil.

${ }^{4}$ Neurosurgery Unit, Universidade Federal de Pernambuco, Recife, Pernambuco, Brazil.

${ }^{5}$ Unimed Recife, Recife, Pernambuco, Brazil.

\section{$\measuredangle$}

Hugo André de Lima Martins Rua Antônio Medeiros Sobrinho, 275, Cabaceira, Surubim-PE, Brasil

hugomt2001@yahoo.com.br

\section{Edited by:}

Marcelo Moraes Valença

Keywords:

Cognitive Behavioral Therapy Facial Pain

Borderline Personality Disorder Lithium Carbonate

Venlafaxine Hydrochloride

\begin{abstract}
Introduction

Borderline personality disorder may be associated with persistent facial pain since its relationship with different pain syndromes has been reported. Persistent idiopathic facial pain is commonly unilateral, pulsating, burning, or profound and challenging for clinicians. Therefore, excluding underlying organic causes by appropriate clinical investigation and complementary tests is essential to diagnose this disease.

Objective

This case report aimed to provide evidence of the relationship between idiopathic persistent facial pain and borderline personality disorder.

Case report

A 24-year-old woman reported severe pain in the left hemiface for ten months, three to six hours per day, five days per week. No abnormalities were found in dental and neurological assessments. A psychiatric evaluation was performed, and the patient met the criteria for borderline personality disorder. Pharmacological treatment consisted of daily lithium carbonate $(900 \mathrm{mg})$ and venlafaxine $(150 \mathrm{mg})$. Weekly sessions of cognitive-behavioral therapy with emotional regulation and tolerance to stress were performed. The patient was evaluated every 30 days and showed improved pain intensity and frequency over six months.

Conclusion

Proper management of borderline personality disorder can modify the evolution of persistent idiopathic facial pain when both pathologies are comorbidities.
\end{abstract}




\section{Introduction}

Dersistent idiopathic facial pain (PIFP) is a chronic disease of unknown etiology. Patients usually complain of unilateral pain affecting the maxillary region, usually described as burning, pulsating, profound, or painful. However, as described in Figure 1, PIFP neither meet criteria for neuralgia nor present objective signs of neurological changes on imaging or clinical examination or dental causes after a detailed investigation. ' Furthermore, few controlled studies demonstrated these patients might present a poor clinical response to antidepressant use (e.g., amitriptyline and nortriptyline) and improved quality of life when associated with psychotherapy. ${ }^{2}$ Association of PIFP and depression, anxiety symptoms, and poor quality of life are well documented in previous studies, although only a few studies about personality traits were performed. ${ }^{2,3}$

A. Facial and/or oral pain fulfilling criteria B and C.
B. Recurring daily for $>2$ hours/day $>3$ months.
C. Pain has both of the following characteristics:
1. Poorly localized and not following distribution of peripheral nerve.
2. Dull, aching, or nagging quality.
D. Clinical neurological examination is normal.
E. Dental cause has been excluded by appropriate investigations.
F. Not better accounted for by another ICHD -3 diagnosis.

Figure 1. Persistent idiopathic facial pain diagnostic criteria (ICHD-3)

A set of stable individual characteristics (i.e., specific functioning patterns) is formed by a set of stable individual characteristics with a personal manner of perceiving oneself, other people, and life events. ${ }^{4}$ However, a personality disorder might be present when this pattern is rigid, pervasive, inflexible, and maladaptive. Personality disorders affect approximately $10 \%$ of the population, with borderline personality disorder (BPD) corresponding to half of this content. ${ }^{5}$

Among all personality disorders, BPD is the most related to painful syndromes. ${ }^{6}$ Table 1 shows the Diagnostic and Statistical Manual of Mental Disorders (DSM-5) diagnostic criteria for BPD (five out of nine criteria are required for diagnosis). BPD is characterized by severe changes in cognition, emotion, behavior, and interpersonal relationships. Self-injurious behaviors are frequent, such as hair pulling, slapping, throwing on the floor, hitting head on the wall, cutting, or scratching. Non-lethal attempt methods sometimes follow suicidal ideation (e.g., excessive medication-taking at once) or violent methods, such as hanging, jumping from high places, throwing themselves in front of automobiles at high speed, and self-harm with knives or firearms. ${ }^{6.8}$

Studies observed a $30 \%$ prevalence rate of BPD among patients with chronic pain. Pain scores are higher in patients with chronic pain and BPD than those patients without BPD. ${ }^{6}$ One study evaluated 777 patients undergoing pain rehabilitation and observed more pain complaints in those with BPD. ${ }^{9}$ In a study evaluating patients with BPD found more painful syndromes (e.g., lumbar pain, temporomandibular joint [TMJ] disorders, and fibromyalgia) in patients with active BPD symptoms than patients with remission of BPD symptoms. ${ }^{10}$
Table 1 Diagnostic and Statistical Manual of Mental Disorders (DSM-5) diagnostic criteria for borderline personality disorder

1. Frantic efforts to avoid real or imagined abandonment (Note: Do not include suicidal or self-mutilating behavior covered in Criterion 5);

2. Pattern of unstable and intense interpersonal relationships characterized by alternating between extremes of idealization and devaluation;

3. Identity disturbance: markedly and persistently unstable self-image or sense of self;

4. Impulsivity in at least two areas that are potentially self-damaging (e.g. spending, sex, substance abuse, reckless driving, binge eating) (Note: Do not include suicidal or self-mutilating behavior covered in Criterion 5);

5. Recurrent suicidal behavior, gestures, threats, or self-mutilating behavior;

6. Affective instability due to a marked reactivity of mood (e.g., intense episodic dysphoria, irritability, or anxiety usually lasting a few hours and only rarely more than a few days);

7. Chronic feelings of emptiness;

8. Inappropriate, intense anger or difficulty controlling anger (e.g., frequent displays of temper, constant anger, recurrent physical fights);

9. Transient, stress-related paranoid ideation or severe dissociative symptoms.

Clinical observations showed that patients with BPD are more intolerant to chronic pain. ${ }^{11}$ Brain imaging studies in BPD demonstrated top-down dysregulation in emotional control ${ }^{12}$ influencing chronic pain signaling. ${ }^{7,12}$ Some neural structures in the fronto-limbic region are also altered in BPD. Studies using functional magnetic resonance imaging demonstrated increased amygdala reactivity and reduced activity in the prefrontal cortex and anterior area of the cingulate gyrus, regions involved in abnormal pain processing. ${ }^{9}$

Therefore, this study aims to report a patient with PIFP and BPD who did not respond to treatments before BPD diagnosis and showed good clinical evolution after proper psychotherapeutic BPD management associated with psychopharmacological treatment for mood stabilization.

\section{Case report}

This study was conducted at the Brain Unity Clinic in Surubim-PE (Brazil) and approved by the human research ethics committee (protocol number 464456921.4.000.5640). This case report concerns a 24-year-old woman with a history of severe pain on the left side of the face for ten months. She reported diffuse pain without specific exacerbation points lasting three to six hours, five days per week. She denied dental procedures before pain onset and attended several dental evaluations because she believed it could be a dental problem. TMJ imaging by magnetic resonance (MRI) and arthroscopy showed nonspecific changes and did not elucidate the cause of pain. She was also evaluated and treated by a neurologist, who did not encounter any abnormalities after a detailed neurological examination of facial sensitivity. Brain MRI was also normal. She used daily $50 \mathrm{mg}$ of amitriptyline, carbamazepine $(600 \mathrm{mg})$, and gabapentin $(900 \mathrm{mg})$ during previous treatments. 
The patient mentioned two events that negatively impacted her life. During childhood (five years old), the first happened when she was run over and hospitalized for several days for medical observation, which psychically affected her. The other event was her mother's absence during baptism, triggering an intense belief of rejection, despite becoming aware of this fact several years later after other people telling her.

In the first evaluation, the patient complained about severe mood instability, intense feelings of emptiness, frequent crisis of unmotivated anger, suicidal ideation, frantic efforts to avoid abandonment, and severe binge eating as emotional regulation (i.e., six out of nine criteria used for BPD diagnosis). During a mental evaluation, the patient was conscious and auto- and allopsychically oriented, anxious, with mood lability, reduced attention and concentration, presented hypomnesia for recent events, organized thinking, and no sensoperceptual alterations.

Pharmacological treatment with lithium carbonate initiated with $300 \mathrm{mg}$ at night, until reaching $900 \mathrm{mg}$ per day, was associated with venlafaxine (initial dose of $37.5 \mathrm{mg}$ per day and final dose of $150 \mathrm{mg}$ after 30 days). Serum lithium dosage was 0.7 millimole per liter during treatment. Weekly sessions of psychotherapy were also indicated with training elements for emotional regulation and tolerance to stress.

Furthermore, four inventories were used to quantify mental changes in a psychiatric evaluation. For Borderline Symptom List 23 (BSL23), an inventory with a list of borderline symptoms, the patient achieved a total score of 44/92 of possible symptoms at initial evaluation and 29/92 after six months of treatment. For Beck Depression Inventory (BDI), the score was 32 at initial evaluation and 13 after six months of treatment. For Beck Anxiety Inventory (BAI), the score was 22 at initial evaluation and nine after six months of treatment. Finally, she achieved an initial score of $6 / 20$ in the Subjective Memory Complaints scale (SMCs), decreasing to $2 / 6$ after six months of treatment. The patient was evaluated every 30 days and improved pain intensity and frequency gradually over six months. Pain intensity was assessed using the eleven-point (0-10) visual analog scale and improved during this period (from eight at initial evaluation to two after six months of treatment) (Figure 2).

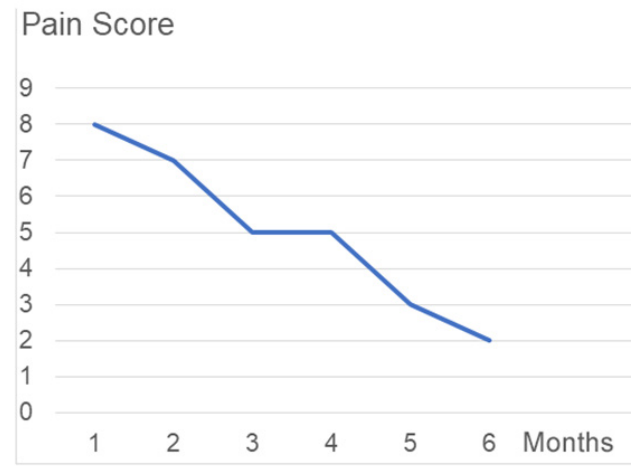

Figure 2. Evolution of pain symptoms on visual pain scale.

\section{Discussion}

This study reports a patient diagnosed with persistent and refractory facial pain investigated for personality disorder. Such comorbidity directly influences PIFP treatment since it changes the therapeutic approach by focusing on pharmacological and psychotherapeutic treatments, most indicated in personality disorders. ${ }^{7}$

Pain is a sensory and emotional experience that may become chronic after six months, leading to loss of function and severe disability. ${ }^{3}$ PIFP is probably the most challenging type of facial pain for clinical management. A multidisciplinary team approach helps identify social, environmental, and intrapsychic factors influencing patient evolution.

Herein, the patient presented a distorted belief of affective bond with her mother because her mother did not attend the baptism. For this reason, the patient developed a trait of insecurity and frequent fear of being abandoned, which is characteristic of BPD patients. ${ }^{7}$ Existential emptiness reported was probably because she thought other people were invalidating her suffering, which is reinforced by lack of findings in complementary exams.

In the past, PIFP was called psychogenic facial pain. A study from the United Kingdom evaluated 150 patients with PIFP and observed that three out of 55 patients excluded were referred to psychiatric hospital admission, and four patients decided to search for psychiatric care at another service. ${ }^{13}$ As $20 \%$ to $25 \%$ of patients admitted to psychiatric hospitals have BPD, it would not be surprising if some of these patients with PIFP also presented severe emotional dysregulation. Another study assessing the personality profile of three patients with PIFP using Cloninger's Temperament and Character Inventory found common characteristics among patients (egocentrism, high levels of insecurity, shame, and harm avoidance), suggesting exaggerated attention to pain and oneself, which may play a fundamental role in chronicity and resistance to treatment. ${ }^{3}$

Depression is present in more than $80 \%$ of patients with BPD. ${ }^{14}$ The clinician must identify the correlation between depression and BPD since treatment with antidepressants alone may not be effective. Anxiety is also commonly found in patients with BPD. BPD and anxiety may not only co-occur but also influence the therapeutic response of each other. ${ }^{15}$ Our patient presented many depressive and anxiety symptoms at the beginning of treatment and improved significantly after six months of follow-up, as shown by the marked decrease in BDI and BAl scores.

Patients with BPD frequently complain about memory problems during daily activities, although this finding is not present in memory tests. ${ }^{16}$ The patient of the present study partially improved subjective memory complaints throughout treatment, as evidenced by SMC. Memory complaints in patients with BPD are probably from the negative self-perception of these patients; therefore, improving affective dysregulation may also improve memory. 
Know about emotional dysregulation is crucial to understand BPD. ${ }^{8}$ The patient described in this study showed severe emotional instability, crises of unmotivated anger, and binge eating. Many patients with BPD manifest emotional dysregulation by alcohol and drug abuse, leading to substance dependence. Other patients manifest this dysregulation in the sexual area (promiscuity conditions), food area (binge eating), or pain area (chronic pain). In this study, the patient manifested characteristics of binge eating and chronic pain.

The interplay between BPD and pain is complex. For example, patients with this disorder are insensitive to acute pain, making superficial skin cuts during self-mutilation episodes. On the other hand, clinical studies show that these patients are more sensitive to chronic pain than individuals without personality disorders. ${ }^{17}$ Therefore, evaluating borderline traits in patients with PIFP is essential because patients with BPD symptoms remission present pain relief and less analgesic use, which are essential for pain chronification. 910

The most important symptom of the studied patient was persistent suicidal ideation, one of the most notable characteristics of BPD. This symptom guided lithium (mood stabilizer with high anti-suicide action) as pharmacological choice ${ }^{18,19}$ associated with venlafaxine (dual-action antidepressant). Moreover, these drugs act in the serotoninergic pathway by decreasing impulsivity, aggressiveness, and the risk of suicide. ${ }^{18}$ However, lithium must be within the therapeutic window due to its high lethality rate. ${ }^{19}$

Psychotherapy was indicated for cognitive restructuring and behavioral change since the patient was diagnosed with a personality disorder. The patient changed her main beliefs regarding her and the outside world using adequate cognitive and behavioral techniques as muscle relaxation and diaphragmatic breathing. Emotion regulation and stress tolerance skills were also provided, contributing to managing inappropriate anger and compulsive eating.

The association between pharmacological treatment for main psychiatric symptoms and psychotherapeutic follow-up probably improved the patient's facial pain. Thus, this study highlights the association between BPD and PIFP. Many PIFP cases may remain unsolved due to the lack of a detailed assessment regarding the meaning of pain of each patient. Ideally, all patients diagnosed with PIFP presenting some degree of refractoriness to usual treatment should be evaluated by a mental health team, increasing treatment efficacy.

Hugo Andre de Lima Martins

https://orcid.org/0000-0002-5216-2074

Bruna Bastos Mazullo Martins

https://orcid.org/0000-0002-6251-992X

Camilla Cordeiro dos Santos

https://orcid.org/0000-0002-5094-7337

Dianilson Jose Pontes

https://orcid.org/0000-0001-9578-5805

Daniella Araújo de Oliveira

https://orcid.org/0000-0002-6013-978X
Marcelo Moraes Valenca

https://orcid.org/0000-0003-0678-3782

\section{References}

1. Headache Classification Committee of the International Headache Society (IHS) The International Classification of Headache Disorders, 3rd edition. Cephalalgia 2018;38(1):1211 Doi: 10.1177/0333102417738202

2. Borges RdS, Kraychete DC, Borges ELG and Melo VMd. Persistent idiopathic facial pain, a diagnosis and treatment of challenge. Case report. BrJP 2018;1(3):279-282 Doi: 10.5935/2595-0118.20180053

3. Christofolleti LM, Oliveira MdFV and Siqueira SRDTd. Personality, coping and atypical facial pain. Case reports. BrJP 2018; 1(1):77-79 Doi: 10.5935/2595-0118.20180016

4. Millon T. Teorias da psicopatologia e personalidade: ensaios e críticas. 2 ed. Rio de Janeiro: Editora Interamericana; 1979. p. 360.

5. Louzã MR and Cordás TA. Transtornos da Personalidade. 2.ed. Porto Alegre: Artmed Editora; 2019. p. 216.

6. Sansone RA and Sansone LA. Chronic pain syndromes and borderline personality. Innov Clin Neurosci 2012;9(1):10-14

7. Martins $\mathrm{H}$. Is borderline personality disorder the cause of chronic headache? Av em Med 2021;1(1):75-76 Doi: 10.52329/ AvanMed. 15

8. Martins H, Martins B, Martins B, Ribas V and Santos A. Borderline and Antisocial Personality Disorders in the Bible. J Psychol Psychother Res 2021;8(1):1 1-21 Doi: 10.12974/23131047.2021.08.2

9. Tragesser SL, Bruns D and Disorbio JM. Borderline personality disorder features and pain: the mediating role of negative affect in a pain patient sample. Clin J Pain 2010;26(4):348-353 Doi: 10.1097/AJP.0b013e3181cd1710

10. Frankenburg FR and Zanarini MC. The association between borderline personality disorder and chronic medical illnesses, poor health-related lifestyle choices, and costly forms of health care utilization. J Clin Psychiatry 2004;65(12):1660-1665 Doi: 10.4088/icp.v65n 1211

11. Harper RG. Personality-guided therapy in behavioral medicine. Personality-guided therapy in behavioral medicine. Washington, DC, US: American Psychological Association; 2004. p. 359.

12. Perez-Rodriguez MM, Bulbena-Cabré A, Bassir Nia A, Zipursky G, Goodman M and New AS. The Neurobiology of Borderline Personality Disorder. Psychiatr Clin North Am 2018;41(4):633650 Doi: 10.1016/i.psc.2018.07.012

13. Feinmann C. Psychogenic facial pain: Presentation and treatment. J Psychosomatic 1983;27(5):403-410 Doi: 10.1016/0022-3999(83)90076-4

14. Beatson J and Rao S. Depression and borderline personality disorder. Med J of Australia 2012;197(11):620-621 Doi: 10.5694/mia 12.11646

15. Bulbena-Cabre A, Perez-Rodriguez MM, Porges S, Bulbena $A$ and Goodman M. Understanding Anxiety in Borderline Personality Disorder. Curr Treat Options Psych 2017;4(2):281294 Doi: 10.1007/s40501-017-0122-0

16. Beblo T, Mensebach C, Wingenfeld K, Rullkoetter N, Schlosser 
$\mathrm{N}$ and Driessen M. Subjective memory complaints and memory performance in patients with borderline personality disorder. BMC Psychiatry 2014;14(1): Doi: 10.1186/s12888-014-0255-2

17. Sansone RA and Sansone LA. Borderline personality and the pain paradox. Psychiatry (Edgmont) 2007;4(4):40-46
18. Berghöfer A. Lithium and suicide. Bmi 2013;347(f4449) Doi: 10.1136/bmi.f4449

19. Benard V, Vaiva G, Masson M and Geoffroy PA. Lithium and suicide prevention in bipolar disorder. Encephale 2016;42(3):234-241 Doi: 10.1016/j.encep.2016.02.006 